\title{
A class of Heisenberg models with the orthogonal dimer ground states
}

\author{
Kazuo Ueda and Shin Miyahara \\ Institute for Solid State Physics, University of Tokyo, \\ 7-22-1 Roppongi, Minato-ku, Tokyo 106-8666, Japan
}

(October 20, 2017)

\begin{abstract}
Extensions of the Shastry-Sutherland model are possible in various ways. In particular, it is possible to construct a natural model in three dimensions which has the exact dimer ground state. Recently found spin gap system $\mathrm{SrCu}_{2}\left(\mathrm{BO}_{3}\right)_{2}$ has this structure. The exchange constants between the layers is expected to be smaller than the intra-layer couplings. However, the exactness of the dimer state for the three dimensional structure is important to understand why magnetic properties of $\mathrm{SrCu}_{2}\left(\mathrm{BO}_{3}\right)_{2}$ are described well by the two dimensional model.
\end{abstract}

75.10.-b, 75.10.Jm, 75.30.Kz

Recently Kageyama et al found a new two dimensional spin gap system $\mathrm{SrCu}_{2}\left(\mathrm{BO}_{3}\right)_{2}$ [1]. The crystal structure of $\mathrm{SrCu}_{2}\left(\mathrm{BO}_{3}\right)_{2}$ is tetragonal and all $\mathrm{Cu}^{2+}$ ions with a localized spin $S=1 / 2$ are located at crystallographically equivalent sites. The two dimensional layers containing the $\mathrm{Cu}^{2+}$ ions are separated by planes of $\mathrm{Sr}^{2+}$ ions. The two dimensional Heisenberg model with the nearest neighbor, $J$, and the next nearest neighbor couplings, $J^{\prime}$ is topologically equivalent to the Shastry-Sutherland model which was found nearly twenty years ago [2].

The model shows a quantum phase transition from the dimer ground state to the Néel ordered state [3]. The critical value for the transition is determined to be $\left(J^{\prime} / J\right)_{c}=0.7 \pm 0.01$. From the magnitude of the spin gap and the Weiss constant the coupling constants for $\mathrm{SrCu}_{2}\left(\mathrm{BO}_{3}\right)_{2}$ are determined as $J=100 \mathrm{~K}$ and $J^{\prime}=68$ $\mathrm{K}$. Thus the ratio between the two coupling constants is very close to the critical value. It is shown that the closeness to the transition point is the origin of the unusual temperature dependence of the susceptibility.

Another novel feature of $\mathrm{SrCu}_{2}\left(\mathrm{BO}_{3}\right)_{2}$ is the magnetization plateaus at one quarter and one eighth of the full $\mathrm{Cu}^{2+}$ moment. Occurrence of the plateaus originates from the nearly localized nature of the triplet excitations in the peculiar lattice structure of the model [3]. Crystallization of the excited triplets at certain magnetization is the origin of the magnetization plateaus.

In this letter we will show that generalization of the Shastry-Sutherland model is possible in several ways. In particular, we can construct several models in three dimensions which have the exact dimer ground states. Interestingly enough, the three dimensional structure of $\mathrm{SrCu}_{2}\left(\mathrm{BO}_{3}\right)_{2}$ has a structure in this class. Although three dimensionality of this compound may not be so important because of small interlayer couplings, it is cer- tainly a helpful fact to understand why the analyses by the two dimensional orthogonal dimer model work so well for this compound.

We start our discussion from two examples in one dimension. The one dimensional version of the ShastrySutherland model is shown in Fig.1(a). The bonds denoted by the thick solid lines define a unique covering of the spins and the dashed lines are the bonds connecting dimers. We use $J$ for the coupling constant in the dimers and $J^{\prime}$ for the inter dimer couplings.

The dimer state is defined by

$$
|\Psi\rangle=\prod_{a}|s\rangle_{a}
$$

where $|s\rangle_{a}$ is the singlet state for the dimer bond specified by index $a$ which runs over all dimer bonds. For this model the dimer state is an exact eigenstate for any $J^{\prime} / J$ and the ground state for small $J^{\prime} / J$ 四. To prove this, let us consider $J^{\prime}$ bonds connecting two dimers, Fig.2(a). It is easy to see that all matrix elements vanish for the dimer state:

$$
J^{\prime}\left(\mathbf{s}_{1}+\mathbf{s}_{2}\right) \cdot \mathbf{s}_{3}|s\rangle_{a}|s\rangle_{b}=0 .
$$

The result holds independent of the magnitude of the spin, $S$.

When we notice that the matrix elements vanish because of the difference in parities between the singlet and the triplet, it is easy to see that the same is true for any dimer pairs when they are orthogonal. For the model of Fig.1(a), the neighboring dimers are orthogonal but in the same plane. A different one dimensional Heisenberg model with the exact dimer ground state may be constructed by making one type of dimers out of the plane, Fig.1(b). In this paper we define the bonds between the orthogonal dimers of this type as $J^{\prime \prime}$ bonds, Fig.2(b). It is straightforward to confirm that all matrix elements of the $J^{\prime \prime}$ bonds vanish:

$$
J^{\prime \prime}\left(\mathbf{s}_{1}+\mathbf{s}_{2}\right) \cdot\left(\mathbf{s}_{3}+\mathbf{s}_{4}\right)|s\rangle_{a}|s\rangle_{b}=0 .
$$

The present model is topologically equivalent to the spin ladders with the diagonal couplings of the same amplitude discussed by Gelfand [5].

It is obvious that any combination of the two types of orthogonal dimers may have the dimer state as the exact ground state. In particular, the dimer state is the exact ground state even for a random distributions of the two dimer configurations when the ratios $J^{\prime} / J$ and $J^{\prime \prime} / J$ are 
sufficiently small. Furthermore, random mixture of spins with different $S$ is possible so long as the spins of each dimer have the same $S$. Distance between the dimers can be different as long as neighboring dimers are orthogonal.

The Heisenberg model for each layer of $\mathrm{SrCu}_{2}\left(\mathrm{BO}_{3}\right)_{2}$ is shown in Fig.3. This model can be considered as a decorated square lattice with orthogonal dimer bonds in the two sublattices. A different model with the exact dimer ground state is obtained by using dimer bonds out of plane in one sublattice. Any combination of dimers along three orthogonal directions is possible so long as the neighboring dimers are orthogonal.

Another type of extension is possible even with only orthogonal dimers in the plane. Let us consider regularly depleted square lattice. For Calcium Vanadates, a series of compounds $\mathrm{CaV}_{n} \mathrm{O}_{2 n+1}$ with $n=2,3,4$ have been synthesized. These Vanadates have layered structures and in each layer the spins of $\mathrm{V}^{4+}$ ions form the structure of the $1 /(n+1)$-th regularly depleted square lattice. By decorating the depleted square lattice we obtain a set of Heisenberg models with the exact dimer ground states for any $S$. Two examples of this class are shown in Fig.4.

Now we proceed to three dimensions. The real three dimensional structure of $\mathrm{SrCu}_{2}\left(\mathrm{BO}_{3}\right)_{2}$ consists of $\mathrm{CuBO}_{3}$ layers and Sr-layers. The $\mathrm{CuBO}_{3}$-layers stack alternately as is shown in Fig.5 [6]. It is clear that the spin- $S$ Heisenberg model of this structure has the exact dimer ground state for small $J^{\prime} / J$ and $J^{\prime \prime} / J$. Again we can construct a broad class of three dimensional Heisenberg models by considering the triad of the orthogonal dimers.

Strictly speaking, for $\mathrm{SrCu}_{2}\left(\mathrm{BO}_{3}\right)_{2}$ the dimers in each plane are not in the same plane: the plane of horizontal dimers is slightly shifted from the plane of vertical dimers. Because of this shift, the distance between $\mathrm{Cu}^{2+}$ ions of the adjacent dimers along the $c$ axis is $3.5930 \AA$ or $4.2325 \AA$. On the other hand, the nearest-neighbor distance and the next-nearest-neighbor distance in each plane are $2.9046 \AA$ and $5.1316 \AA$. Actually the $\mathrm{Cu}^{2+}-\mathrm{Cu}^{2+}$ distances between the planes are shorter than the next-nearest-neighbor distance in the plane. In spite of this fact, we expect that $J^{\prime}$ is much bigger than $J^{\prime \prime}$ since the dominant path of the superexchange is through the molecular orbital of $\mathrm{BO}_{3}$. On the other hand, $\mathrm{Sr}^{2+}$ ion has a closed shell. Therefore the two dimensional orthogonal dimer model is a good starting point for $\mathrm{SrCu}_{2}\left(\mathrm{BO}_{3}\right)_{2}$. However the exactness of the dimer ground state for the three dimensional model is the reason why magnetic properties of $\mathrm{SrCu}_{2}\left(\mathrm{BO}_{3}\right)_{2}$ are described well by the two dimensional model.

It is our great pleasure to thank Dr. H. Kageyama for many helpful discussions. We have also enjoyed illuminating discussions with Dr. B.S. Shastry.
[1] H. Kageyama, K. Yoshimura, R. Stern, N.V. Mushnikov, K. Onizuka, M.Kato, K. Kosuge, C.P. Slichter, T. Goto, and Y. Ueda: Phys. Rev. Lett. (1999) in press.

[2] B.S. Shastry and B. Sutherland: Physica B 108 (1981) 1069.

[3] S. Miyahara and K. Ueda: Phys. Rev. Lett. (1999) in press.

[4] N.B. Ivanov and J. Richter: Phys. Lett. A 232 (1997) 308.

[5] M.P. Gelfand: Phys. Rev. B 43 (1991) 8644.

[6] H. Kageyama: private communication.

(a)

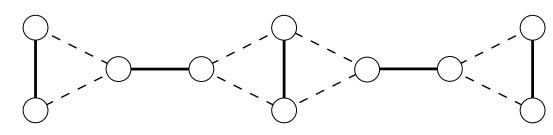

(b)

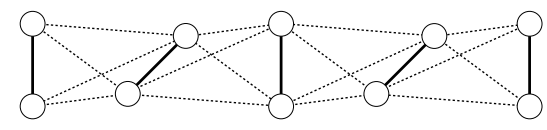

FIG. 1. Two examples of one dimensional orthogonal dimer models.

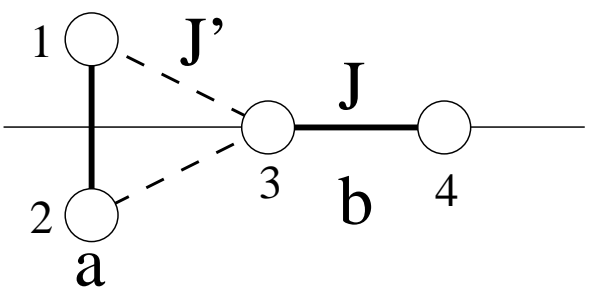

(a)

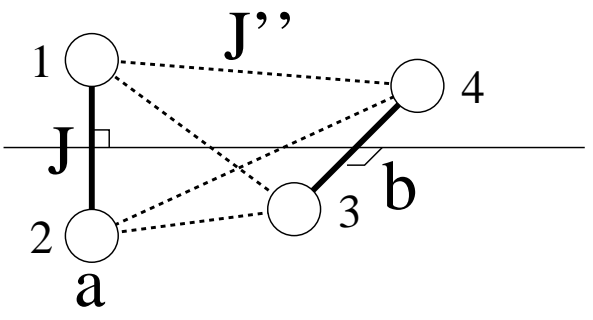

(b)

FIG. 2. Two configurations of orthogonal dimers. 


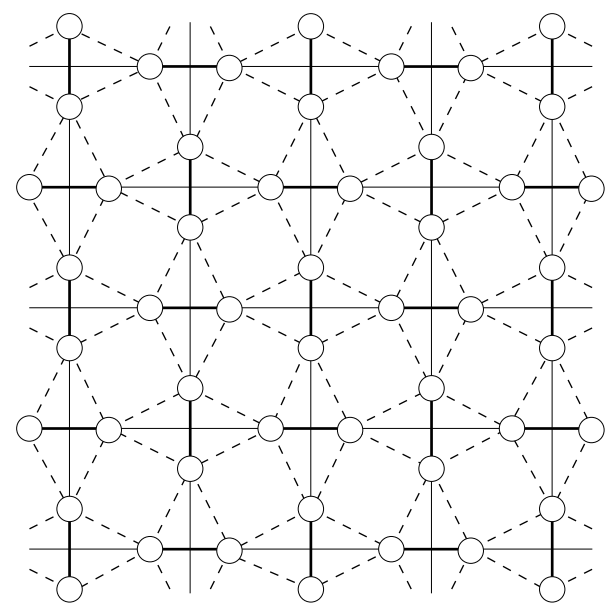

FIG. 3. The model for $\mathrm{SrCu}_{2}\left(\mathrm{BO}_{3}\right)_{2}$ : Two dimensional orthogonal dimer model.
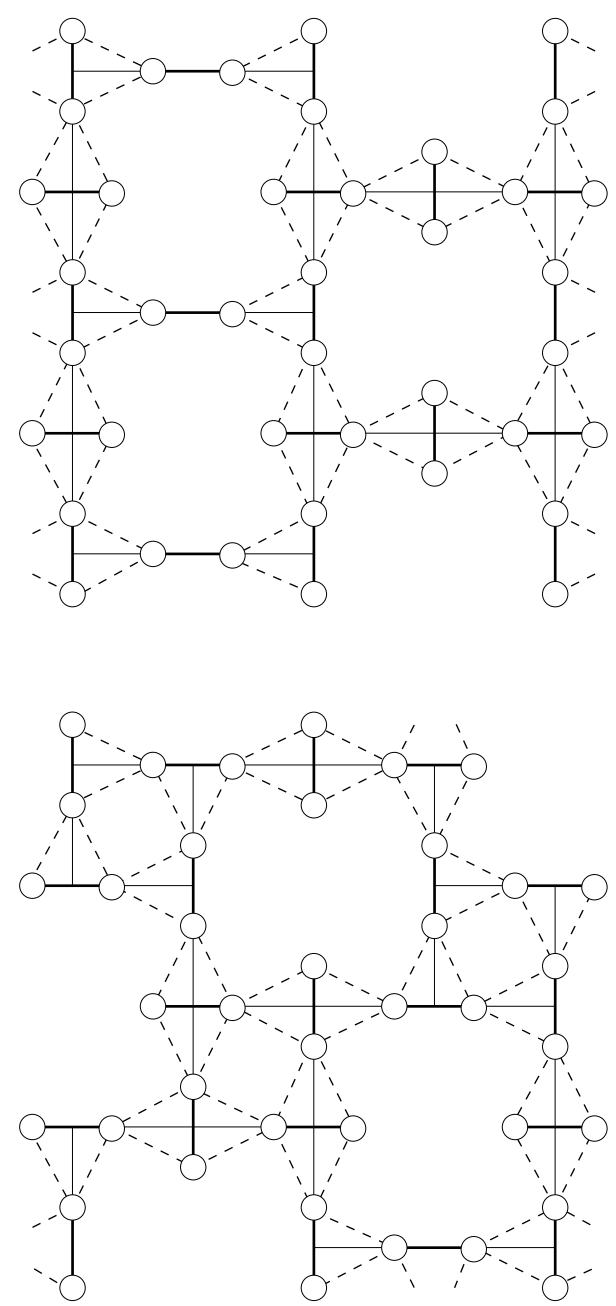

FIG. 4. Two examples of the regularly depleted square lattices with decoration of orthogonal dimers.

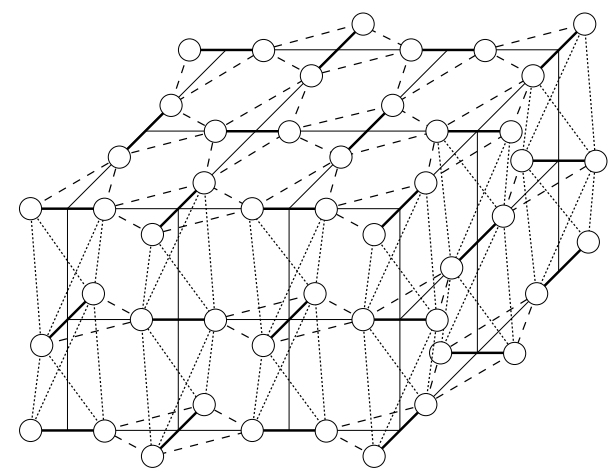

FIG. 5. Three dimensional orthogonal dimer model. 